\title{
Assistência de enfermagem aos pacientes em cuidados paliativos
}

\author{
Nursing care for patients in palliative care \\ Cuidados de enfermería para pacientes en cuidados paliativos
}

Recebido: 15/05/2021 | Revisado: 23/05/2021 | Aceito: 26/05/2021 | Publicado: 01/06/2021

Silvana Carloto Andres
ORCID: https://orcid.org/0000-0001-6726-7947
Universidade Federal de Santa Maria, Brasil
E-mail: silvana.andres@yahoo.com.br
Liane Bahú Machado
ORCID: https://orcid.org/0000-0002-1356-373X
Universidade Federal de Santa Maria, Brasil
E-mail: lianemachado61 @ gmail.com
Fábio Piazer Franco
ORCID: https://orcid.org/0000-0001-8179-177X
E-mail: fabiopiazerfranco@ @otmail.com
Daniel Santos dos Santos
ORCID: https://orcid.org/0000-0002-6657-7924
Secretaria Municipal de Cacequi, Brasil
E-mail: danielsantosdoss@ hotmail.com
Rafaella França Torres
ORCID: https://orcid.org/0000-0002-9291-5341
Hospital São Roque - Faxinal do Soturno, Brasil
E-mail: rafaellaftorres@ hotmail.com
Silvana Urrutia Pedroso

\section{Resumo}

Os Cuidados Paliativos apresentam competências relacionadas ao cuidado físico, psíquico, social e espiritual de pacientes que enfrentam doenças que ameacem suas vidas. Isso pode e deve ser integradas precocemente ao cuidado para o controle da doença, e diminuição dos sintomas, gerando assim melhoria na qualidade de vida. Esta pesquisa tem o objetivo de descrever o conhecimento do profissional enfermeiro na assistência para melhoraria da qualidade de vida, alivio da dor e sofrimento dos pacientes em cuidados paliativos. Trata-se de uma revisão de literatura do tipo narrativa com abordagem qualitativa, a busca ocorreu de setembro a dezembro de 2020 - incluiu fontes impressas e on-line gratuitas, a exemplo de manuais, protocolos, artigos e periódicos: Literatura Latino-Americana e do Caribe em Ciências da Saúde (Lilacs), Bibliográficos Especializada na Área de Enfermagem do Brasil (BDENF) e Medical Literature Analysisand Retrieval System Online (Medline). Para operacionalizar a busca foi utilizado o recurso: "CUIDADOS PALIATIVOS" [Descritor de assunto] and "ENFERMAGEM" [Descritor de assunto] nas bases de dados citadas. O enfermeiro tem um papel fundamental para a promoção do CP, como na aceitação do diagnostico e auxilio para conviver com a doença, prestando assistência integral ao usuário e a todos envolvidos com o doente.

Palavras-chave: Cuidados Paliativos; Enfermagem; Cuidados.

\begin{abstract}
Palliative Care has competencies related to the physical, psychological, social and spiritual care of patients who face diseases that threaten their lives. This can and should be integrated early into the care for the control of the disease, and reduction of symptoms, thus generating an improvement in the quality of life. This research aims to describe the knowledge of the professional nurse in assistance to improve the quality of life, relieve pain and suffering of patients in palliative care. It is a narrative-type literature review with a qualitative approach, the search took place from September to December 2020 - it included free printed and online sources, such as manuals, protocols, articles and periodicals: Latin American Literature and of the Caribbean in Health Sciences (Lilacs), Bibliographic Specialized in the Brazilian Nursing Area (BDENF) and Medical Literature Analysisand Retrieval System Online (Medline). To operationalize the search, the resource was used: "PALLIATIVE CARE" [Subject descriptor] and "NURSING" [Subject descriptor] in the aforementioned databases. The nurse has a fundamental role in the promotion of PC, as in the acceptance of the diagnosis and help to live with the disease, providing comprehensive assistance to the user and everyone involved with the patient.
\end{abstract}

Keywords: Palliative Care; Nursing; Care. 


\begin{abstract}
Resumen
Los Cuidados Paliativos tienen competencias relacionadas con el cuidado físico, psicológico, social y espiritual de los pacientes que enfrentan enfermedades que amenazan su vida. Esto puede y debe integrarse precozmente en la atención para el control de la enfermedad y la reducción de los síntomas, generando así una mejora en la calidad de vida. Esta investigación tiene como objetivo describir los conocimientos del enfermero profesional en la asistencia para mejorar la calidad de vida, aliviar el dolor y el sufrimiento de los pacientes en cuidados paliativos. Se trata de una revisión de literatura de tipo narrativo con enfoque cualitativo, la búsqueda se realizó de septiembre a diciembre de 2020, incluyó fuentes gratuitas impresas y en línea, como manuales, protocolos, artículos y revistas: Literatura Latinoamericana y Ciencias de la Salud del Caribe (Lilacs ), Bibliográfico Especializado en Enfermería en Brasil (BDENF) y Sistema de Análisis y Recuperación de Literatura Médica en Línea (Medline). Para hacer operativa la búsqueda se utilizó el recurso: "PALIATIVE CARE" [Asunto descriptor] y "ENFERMERÍA" [Asunto descriptor] en las bases de datos citadas. El enfermero tiene un papel fundamental en la promoción de la CP, como en la aceptación del diagnóstico y la ayuda a convivir con la enfermedad, brindando una asistencia integral al usuario y a todos los involucrados con el paciente.
\end{abstract}

Palabras clave: Cuidados Paliativos; Enfermería; Cuidado.

\title{
1. Introdução
}

O cuidado paliativo $(\mathrm{CP})$ visa à promoção da qualidade de vida de pacientes e seus familiares, através da avaliação precoce, controle de sintomas físicos, sociais, emocionais e espirituais, no contexto de doenças que ameaçam a continuidade da vida. Estas necessitam ser unidos ao cuidado que busca o controle da doença. A demanda em Cuidados Paliativos é crescente em todo o mundo devido ao envelhecimento populacional (ANCP, 2020). Os órgãos nacionais e internacionais de saúde enfatizam cada vez mais a necessidade de criação de políticas baseadas em evidências, reconhecendo o paliativismo como essencial no cuidado nos serviços de saúde (Brasil, 2020).

CP é um termo recente, e é aplicado para o conceito de cuidados para designar a ação conjunta multiprofissional, a pacientes sem chances terapêuticas/medicamentosas de cura, relacionado ao tratamento convencional. O termo paliativo é originado do latim palliun que significa proteção, ou seja, proteger aqueles aos quais a medicina curativa não consegue curar (Silva et al., 2017).

Em 31 de outubro de 2018, o Ministério da Saúde publicou a resolução $n^{\circ} 41$, que normatiza a oferta de cuidados paliativos como parte dos cuidados continuados no Sistema Único de Saúde (SUS). A resolução recomenda que seja identificada as prioridades da pessoa doente quanto ao cuidado e tratamento que receberá. A resolução define ainda, que os $\mathrm{CP}$ devam estar disponíveis em toda a rede de saúde: atenção básica, domiciliar, hospitalar incluindo a rede de urgência e emergência (Brasil, 2018).

Para que o CP abranja todas as áreas da vida do paciente, há maior necessidade de uma equipe multiprofissional, para melhor assistência a esse paciente, com planejamento e um consenso no método terapêutico a ser utilizado (Santos et al., 2019). Tais cuidados se iniciam com o diagnóstico da doença e se estendem até o luto é necessária uma equipe multiprofissional qualificada, com preparo para que haja interação e muita dedicação aos pacientes e sua família (Brasil, 2008).

A enfermagem como membro integrante da equipe multiprofissional paliativa, em um serviço pode atuar tanto no plano técnico terapêutico, ou na gerência de cuidados quanto na compreensão da natureza humana, direcionando a atenção para as necessidades holísticas do paciente e da família, planejando e implementando ações que permitam ao indivíduo o máximo controle sobre sua vida e doença (Andrade et al., 2019).

Em relação à assistência que prestada ao paciente destaca-se a conversa como um método importante, principalmente quando não há mais chances de cura. A comunicação age associada diretamente com a humanização, possibilitando ao enfermeiro e o paciente/familiares, de maneira holística, uma positiva troca de conhecimentos e experiências na qual o objetivo principal também é preservar a saúde mental do paciente para que ele seja capaz de manter-se equilibrado e calmo, buscando tornar esse momento o mais leve possível (Pacheco et al., 2020). 
Esta pesquisa teve como objetivo descrever o conhecimento do profissional enfermeiro na assistência para melhoraria da qualidade de vida, alivio da dor e sofrimento dos pacientes em cuidados paliativos.

\section{Metodologia}

Este artigo utilizou-se do método de revisão narrativa da literatura, método cujo objetivo principal é a reunião e síntese de resultados sobre o tema, apresentando e potencializando a construção de conhecimentos para possibilitar a incorporação de evidências na prática clínica. A revisão narrativa das demais revisões por permitir a inclusão de pesquisas de métodos diversos buscando um conhecimento mais amplo da literatura para conhecer e entender o fenômeno estudado (Medeiros et al., 2015).

A busca ocorreu de setembro a dezembro de 2020 -Para a seleção dos estudos foi realizada uma busca online em periódicos e nas bases de dados LILACS, SCIELO, BDENF Para operacionalizar a busca foi utilizado o recurso: "CUIDADOS PALIATIVOS" [Descritor de assunto] and "ENFERMAGEM" [Descritor de assunto] nas bases de dados citadas anteriormente. Na segunda fase, foi selecionada toda a bibliografia considerada relevante e relacionada ao objetivo proposto. Foram incluídos protocolos, manuais e artigos nos idiomas português e inglês publicados no período de 2015 a 2020. Serviram como critérios de exclusão: a inacessibilidade do texto completo por via eletrônica ou impressa; trabalhos escritos em outras línguas; e trabalhos que não contemplavam a assistência de enfermagem e cuidados paliativos em pacientes adultos ou os desfechos relacionados ao tema. O conteúdo pesquisado foi categorizado de acordo com os critérios de inclusão, possibilitando a posterior construção dos materiais.

Para operacionalização desta revisão, foi utilizado a busca, avaliação crítica e a síntese das evidências disponíveis do tema investigado. E para a composição final dos artigos, foram estabelecidas duas etapas: na primeira, foi realizada análise dos títulos e resumos, confirmando se eles contemplavam a pergunta norteadora desta investigação e os critérios de inclusão. Na segunda etapa, foi efetivada a leitura integral exploratória dos artigos restantes, que que permitiu evidenciar as principais convergências encontradas, as quais foram sintetizadas, agrupadas, categorizadas e analisadas a partir da análise de conteúdo de Minayo que compreendem: a pré-análise, a exploração do material, o tratamento dos resultados obtidos e a interpretação (Minayo, 2010).

\section{Resultados e Discussão}

A busca resultou em 55 artigos, que após critérios de exclusão foram utilizados 26 artigos elegíveis, para a construção, mas após filtragem, foram consolidados 15 artigos para compor o presente estudo e divididos em duas categorias, que são elas: Cuidados paliativos: definição, princípio e legislação; Dificuldades de Implantar os Cuidados Paliativos no Brasil; e; Enfermagem no cenário dos cuidados paliativos.

\section{Cuidados paliativos: definição, princípio e legislação no Brasil}

Em 1947, Cicely Saunders, criou o conceito de dor total, que explica a ideia de que o cuidado à pessoa só terá eficácia se for levando em consideração suas dimensões sócias cultural, física, psíquica e espiritual, ressaltando a prática multiprofissional em cuidados paliativos (Manchola et al., 2016).

O CP tem o objetivo suavizar a sintomatologia da doença que é progressiva e incurável, essa associação iniciou na primeira metade do século XIX com trabalhos de Jeanne Garnier. Ligado a esse tipo de cuidado e ao fenômeno mundial do envelhecimento populacional, aumento de números de pacientes com diagnóstico de câncer, patologias incuráveis e o avanço das doenças crônicas e degenerativas em estado avançado ou terminal, colocou o CP como urgência no cuidado da 
coletividade, requerendo investimentos para que seja ofertado pelos sistemas de saúdes, inclusive no sistema único de saúde brasileiro, para atender as necessidades da população (Gaspar \& Silva, 2017).

Em 2002, a Organização Mundial de Saúde (OMS), definiu que os CP, como uma abordagem de tratamento que contribui na melhora a qualidade de vida de pacientes e familiares perante as doenças que ameacem a continuidade da vida. Assim o CP, deve agrupar as habilidades de uma equipe multiprofissional para amparar o paciente e seus familiares a se adaptarem às mudanças de vida, promovendo a reflexão para o enfrentamento da condição da doença, prevenção alívio do sofrimento, avaliação e tratamento adequados dos problemas físicos, psicossociais, e a possibilidade irreversível de morte de modo a estabelecer um cuidado que não abrevie a morte, nem a prolongue, porém contribua para uma melhoria da qualidade de vida nesse momento (Costa et al., 2016).

Não existe um único local em que se podem realizar cuidados paliativos, o mais indicado é onde o paciente que necessita desse cuidado estiver, ou seja, no domicílio, no hospital, instituição de longa permanência ou qualquer outro serviço de saúde (Brasil, 2020).

Muitos dos pacientes permanecem internados por longos períodos, longe de familiares e da sua residência, na maioria das vezes submetida a procedimentos invasivos e dolorosos desnecessários por falta de conhecimento e treinamento da equipe multiprofissional que os assiste, proporcionando mais dor e desconforto, ao contrário do que preconiza esse modelo de cuidado.

O CP possui uma ligação direta com os princípios da bioética, que visa destacar a autonomia e os valores de cada ser humano diante de cada tratamento, respeitando a liberdade de cada paciente, com todos os procedimentos realizados no paciente discutido previamente em equipe e com familiares (Andrade et al., 2016).

No cenário de saúde e na assistência de enfermagem, o enfermeiro se depara com o $\mathrm{CP}$ em diferentes ambientes da prática de enfermagem, apesar disso, a prática deste cuidado pode ser prejudicada por falta de formação específica ou de experiências e vivências que proporcionem o aprendizado e viabilize uma prática assistencial de qualidade, proporcionando qualidade de vida, alívio do sofrimento e da dor na proximidade da morte para o paciente (Silveira et al., 2016).

$\mathrm{O}$ âmbito do CP é uma esfera da assistência que está em constante construção, a enfermagem vem ofertando inúmeros cuidados primordiais para a população nessas condições. Através da padronização de linguagem o profissional de enfermagem precisa possuir um raciocínio clínico sagaz a fim de reconhecer as respostas humanas de grande complexidade que esse usuário possa requerer, com intuito de direcionar o cuidado prestado e as orientações oferecidas o mais próximo possível de suas necessidades (Passarelles, Rios, Santana 2019).

Neste caso em especial a equipe de enfermagem, são os profissionais que estão a maior parte ao lado dos pacientes prestando cuidados curativos ou paliativos, que tem por objetivo trazer dignidade humana ao processo de morte ao paciente, que distingue de uma assistência comum, intervencionista e curativista, a enfermagem pode valer-se quando possível também de medidas não farmacológicas como o uso de escalas e ainda uso das Praticas Integrativas e Complementares em Saúde.

\section{Dificuldades de Implantar os Cuidados Paliativos no Brasil}

No Brasil, o CP, ainda se compõe como uma modalidade emergente de assistência em terminalidade de vida, esse modelo de cuidado consiste em aprendizados direcionadas ao paciente, afastando o conceito curativista, entende-se que a doença nesse momento já está progressiva e irreversível e não responsiva ao tratamento clinico estabelecido usualmente. Com isso, os profissionais devem proporcionar uma assistência adequada, individualizada e integral, visando conforto nos momentos finais de vida (Silveira et al., 2016).

No intuito de melhorar, qualificar e humanizar a assistência a pacientes crônicos ou portadores de doenças sem cura, o $\mathrm{CP}$, passou a integrar a Política Nacional de Humanização, que embora ainda existam fatores que dificultam à prática do 
paliativismo, como a dificuldade de acesso aos medicamentos opioides, a insuficiência de serviços e programas especializados em CP, carência de uma disciplina específica na formação de profissionais de saúde nos cursos de graduação levando ao despreparo na atuação profissional, causando uma grande lacuna na aplicação do CP nos pacientes que necessitam desse tipo de cuidado (Costa et al., 2016).

De acordo com a história, os profissionais de saúde foram educados com o conceito de que havia necessidade de se fazer o tratamento terapêutico pela cura ou melhora de um paciente. (ANCP, 2020). Assim, o adoecimento causa nos pacientes, profissionais e familiares/cuidadores um intenso querer de cura, e que nesse processo se alcancem possibilidades de tratamento e cura do paciente doente. Contudo, quando a resposta positiva não é obtida, os sujeitos envolvidos neste processo, acabam por sentir inúmeros sentimentos negativos, frustrações e impotência diante da probabilidade da perda e/ou da continuação da vida (Queiroz et al., 2018).

No momento que a enfermidade progride e a morte se aproxima, são realizadas ações de enfrentamento pelos familiares para entender as dificuldades vivenciadas e as que estão por vir, é fundamental uma atenção ao suporte emocional, espiritual e social para o paciente e sua família, enfrentem o processo com dignidade, tudo isso acontece envolto a um misto de sentimentos e condutas que precisam ser compreendidos e acompanhados pelos profissionais de saúde, o CP é inserido como meta para assegurar a melhor qualidade de vida possível ao paciente e sua família durante todo o período de tratamento, de morte e luto (Andrade et al., 2019).

A mudança do paradigma entre a cura e o CP é um método que depende dos profissionais na área, e das instituições acadêmicas que são responsáveis pela formação destes profissionais, incluindo essa temática nas disciplinas de graduação de todas as áreas da saúde, bem como preparando o profissional para essa interface do cuidado. Portanto, é necessário que seja uma prática alicerçada na capacitação profissional conduzida por princípios científicos baseada em artigos, publicações, protocolos e normativas de saúde que abordem esse tema (Baliza et al., 2015).

No Brasil, as atividades relacionadas a Cuidados Paliativos ainda necessitam ser regularizadas na forma de lei, principalmente entre os profissionais de saúde, gestores hospitalares e poder judiciário, é preciso que os serviços de saúde estabeleçam protocolos e normativas para esse cuidado, promovendo e aplicando esses cuidados, para que não se confunda o atendimento paliativo com eutanásia (Brasil, 2020).

É necessário ainda que haja uma conscientização coletiva da população brasileira sobre os $\mathrm{CP}$, essa conscientização é essencial para que o sistema de saúde transforme suas abordagens aos pacientes que estão com a ameaça de prosseguimento de suas vidas, o paliativismo é uma necessidade de saúde pública, além de uma necessidade humanitária (Brasil, 2020).

\section{A enfermagem no cenário dos cuidados paliativos}

Atualmente existe um grande aumento na cronicidade de doenças, proporcionando maior tempo de vida populacional, influenciando diretamente na mudança dos tipos de cuidados existentes para o alivio da dor ou conforto do paciente.

No entanto, o conceito "cuidados paliativos" é utilizado para designar a ação de uma equipe multiprofissional (qual inclui o enfermeiro), à pacientes fora de possibilidades terapêuticas de cura. Porém estudos mostram que percebendo que frente à impossibilidade de cura, o profissional enfermeiro manifesto o respeito pela vida do paciente ao se comprometer em protegêlo diante de tal vulnerabilidade, pois o CP começa quando o cuidado curativo não tem mais o propósito principal (França \& Cardoso, 2017).

Quando relacionado à diminuição da dor dos sintomas físicos, a enfermagem necessita aprender a interpretar e compreender não somente as queixas verbais, mas as expressões corporais e faciais, quando possível pode-se utilizar escalas especificas para avaliação da dor, e interpretação dos sinais fisiológicos, mas sempre com muita cautela para o ponteiro da obstinação terapêutica, pois são técnicas que podem se tornar repetitivas na rotina do paciente (Franco et al., 2017). 
É necessário que o enfermeiro direcione suas orientações ao paciente sobre o $\mathrm{CP}$, instigue as respostas ao pensamento alheio, com objetivo de desconectar o paciente de sua doença por instantes e estimular e encorajar o paciente mostrando-se estar ao lado do mesmo em todos os momentos, para que este se sinta acolhido e valorizado (Rapanos, Oliveira, Ferreira 2020).

$\mathrm{O}$ assunto paliatividade é marginalizada na de formação da enfermagem, estando presente apenas nas discussões que envolvem o adoecimento e morte, esse distanciamento do tema nos currículos pode estar relacionado à aproximação tardia desta filosofia da realidade brasileira, porém esse distanciamento pode ocasionar/ levar o enfermeiro a ter dificuldades em entender e aplicar os cuidados paliativos na assistência em saúde ao paciente necessitado desse cuidado (Ávila et al., 2017).

É evidente que o enfermeiro desempenha uma assistência de grande importância, pois possui contato com o paciente diariamente e diretamente atentando para suas necessidades e aprimorando as mesmas. Portanto, é possível assegurar que a enfermagem é uma peça chave no que diz respeito ao tratamento paliativo dos pacientes, pois a assistência ofertada pela enfermagem não abrange apenas o sofrimento físico, mas inclusive o sofrimento social e espiritual do paciente e de seus familiares (Perondi, et al., 2019).

É notória a necessidade dos profissionais de saúde buscarem cuidados de promoção, prevenção de agravos e de recuperação da saúde, bem como valorizarem um morrer digno, assumindo que a morte não deve ser uma inimiga a se vencer, mas um evento natural integrante da vida (Silva et al., 2017).

Procedimentos terapêuticos sem finalidade curativa dão conforto ao paciente, mas no estado de terminalidade, em que não há probabilidade de cura, a tendência é a diminuição de procedimentos curativista e a inserção dos procedimentos paliativista. Por isso, o cuidar respeita valores e crenças dos pacientes, atuando de modo ético e empático, preocupando-se não apenas com ações técnicas, mas com o saber e o fazer de forma humanizada (França \& Cardoso, 2017).

O enfermeiro como parte da equipe interdisciplinar de CP exerce um papel fundamental na promoção e acesso de cuidados para o paciente sem probabilidades de cura, necessitando ser respaldos em princípios éticos que valorizam a autonomia do paciente como um dos pontos fundamentais à busca da dignidade dos cuidados prestados pela enfermagem (Andrade et al., 2016).

As situações de final de vida colocam os enfermeiros diante do sofrimento da família, e nesta situação, trabalhar com as famílias é importante, especialmente quando o paciente está fora de possibilidades de cura.

O enfermeiro é considerado um profissional que está presente no atendimento de pacientes com doença em fase de terminalidade, realizando procedimentos que confortam e aliviam a dor e proporciando bem-estar, cuidar e apoiar o paciente e os familiares em paliatividade requer do profissional preparo técnico-científico e emocional. A mudança de paradigma, da cura do paciente para o do cuidado e alívio do sofrimento do paciente e da família, é um processo lento e depende das iniciativas individuais dos enfermeiros (Baliza et al., 2015).

O enfermeiro é o que permanece mais tempo ao lado do paciente, deve se colocar na posição de facilitador da promoção da qualidade de vida do paciente sem possibilidades terapêuticas tendo como base os princípios da Bioética: o princípio da autonomia, da beneficência, da não maleficência e da justiça (Andrade et al., 2016).

A enfermagem é parte fundamental na equipe de cuidados paliativos, precisando proporcionar educação em saúde de maneira clara e objetiva, com ações práticas que visem o alivio da dor e do sofrimento, gerando o bem-estar e qualidade de vida do paciente. A assistência de enfermagem no $\mathrm{CP}$ deve considerar o paciente um ser singular, completo e multidimensional, tornando o cuidado, integral e humanizado, só é possível quando o enfermeiro faz uso de diversidades de comunicação verbal e não verbal (Santos et al., 2017).

O enfermeiro busca amenizar a dor e sofrimento do paciente oferecendo uma assistência humanizada e individualizada, o enfermeiro deve observar se essa assistência trará benefícios e efeitos através do diálogo, deixando visível que embora as implicações não sejam de cura, mas de melhoria no bem-estar e na qualidade de vida no restante de vida do 
paciente. Procurar entender a individualidade de cada paciente através de tecnologias leve e duras disponíveis em saúde são benefícios que podem ser adotadas pela equipe de enfermagem (Gomes et al., 2015).

A enfermagem no processo de morte está acompanhada diariamente da aflição e angústia destes pacientes e de seus familiares, em alguns momentos, a enfermagem pode se sentir impotente, ou despreparada, pois muitas vezes, apenas aprendeu a cuidar para cura. (Silva et al., 2017).

$\mathrm{O}$ enfermeiro é um dos profissionais de saúde que mais compõem a equipe de $\mathrm{CP}$ e tem como principal propósito garantir de maneira séria a qualidade de vida para os pacientes nessas condições, os quais estão/são acometidos por doenças que ameacem sua vida e causem angústia aos seus familiares. Nesse sentido, o enfermeiro precisa agir procurando prevenir e amenizar o sofrimento através da avaliação, identificação precoce, e controle da dor em todos os aspectos (Leite et al., 2020).

$\mathrm{O}$ enfermeiro que atua em CP, em relação a esta e demais atribuições que lhe pertencem, age como um solucionador, então, tem por papel avaliar toda necessidade não completada, e sugerir soluções para elas. As necessidades psicossociais e espirituais não deixem de ser uma delas, então devem ser propostos e executados suportes para estas (Santos et al., 2019).

Os cuidados de enfermagem vão muito além de apenas intervenções técnicas, como por exemplo, a administração de medicamentos, curativos, avaliações clínicas entre outros, tendo o foco do seu atuar direcionado para o ser humano e não para a doença, procurando agir e intervir no controle da dor e do sofrimento nas circunstâncias biopsicossocial e espiritual dos pacientes e de seus familiares (Leite et al., 2020).

\section{Considerações Finais}

A evidência da morte é uma constante em nossas vidas, é inevitável e faz parte do ciclo da vida de todo ser humano, mas dificilmente é aceitável, principalmente quando envolve os sentimentos de todo o grupo familiar, neste sentido o trabalho em equipe multi de saúde facilita a abordagem através de informações e orientações mais adequadas à família e ao paciente possibilitando uma maior capacidade de oferecer conforto e alivio do sofrimento.

Essa temática é de importância na teoria é na prática. Sugere-se que cada vez mais esse assunto seja pesquisado e publicando, para que possa atingir a maior parte da população possível.

Ainda salienta-se a necessidade da inclusão de cuidados paliativos na formação acadêmica dos profissionais de saúde, favorecendo o conhecimento do tema, para o profissional lidar com a morte e o morrer, sendo incluindo espaços nas universidades para debates sobre a temática, ou ainda que esse espaço sirva para atender pessoas que passam por esse processo seja eles familiares ou pacientes.

\section{Referências}

ANCP - Academia Nacional de Cuidados Paliativos. São Paulo (2020). Atlas dos Cuidados Paliativos no Brasil 2019. https://apiwordpress.paliativo.org.br/wp-content/uploads/2020/05/ATLAS_2019_final_compressed.pdf

Andrade, C. G et al (2016). Cuidados Paliativos e Bioética: Estudo com enfermeiros assistenciais. Rev online de pesquisa: Cuidado é fundamental; 2 (8). p 17.

Andrade, G. B. D. et al. (2019). Cuidados Paliativos e a Importância da Comunicação entre o Enfermeiro e Paciente, Familiar e Cuidador. Revista Online de Pesquisa; 11. p 1-5.

Ávila, F. B. P. et al (2017). Cuidados Paliativos: Refletindo As Influências e Contrassensos da Filosofia de Saunders na Formação do Enfermeiro; 8 (1).

Baliza, M. F. (2015). Fatores que influenciam os enfermeiros de Unidades de Terapia Intensiva nas decisões de final de vida. Rev Escola Enfermagem da USP; 49 (4). p 572-579.

BRASIL (2018). Resolução $n^{\circ} 41$, de 31 de outubro de 2018. Dispõe sobre as diretrizes para a organização dos cuidados paliativos, à luz dos cuidados continuados integrados, no âmbito Sistema Único de Saúde (SUS). /asset_publisher/Kujrw0TZC2Mb/content/id/51520746/do1-2018-11-23-resolucao-n-41-de-31-de-outubro-de-2018-51520710 
Research, Society and Development, v. 10, n. 6, e55910616140, 2021

(CC BY 4.0) | ISSN 2525-3409 | DOI: http://dx.doi.org/10.33448/rsd-v10i6.16140

BRASIL. (2008). Ministério da Saúde. Instituto Nacional do Câncer. Ações de enfermagem para o controle do câncer: uma proposta de integração ensinoserviço. 3. ed. atul. ampl. Rio de janeiro: INCA. Disponível em: http://www.inca.gov.br/enfermagem/docs/ficha_tecnica.pdf .

Costa, Á. P et al. (2016). Formação em cuidados paliativos: experiência de alunos de medicina e enfermagem. Interface - Comunicação, Saúde, Educação; 20 (59). p 1041-52.

França, K.H. D. P; \& Cardoso, M.V.N. (2017). O Aprendizado Para A Prática Do Cuidado Paliativo Em Oncologia Sob A Ótica Dos Enfermeiros. Dissertação (mestrado) - Universidade Federal do Rio de Janeiro, Escola de Enfermagem Anna Nery, Programa de Pós-Graduação em Enfermagem.

Franco, H.CP et al. (2017). Papel da enfermagem na equipe de cuidados paliativos: a humanização no processo da morte e morrer. Rev gestão \& saúde, 17 (2): $48-61$.

Gaspar, R. B; \& Silva, M. M. (2017). O Gerenciamento Do Cuidado De Enfermagem Para Defesa Da Autonomia Dos Idosos Em Terminalidade Da Vida. Dissertação (Mestre em Enfermagem) Universidade Federal do Rio de Janeiro.

Gomes, C. Y. O. S et al. (2015). O Enfermeiro e os Cuidados Paliativos Prestados a Pacientes Oncológicos Terminais. SANARE - Revista de Políticas Públicas; (14). p 71-81.

Leite, A. C. et al. (2020). Assistência de enfermagem nos cuidados paliativos ao paciente idoso em unidade de terapia intensiva. Rev Braz. J. of Develop; (6)12. p 102261-102281.

Manchola, C. et al. (2016). Cuidados paliativos, espiritualidade e bioética narrativa em unidade de saúde especializada. Rev. Bioét., Brasília; 24 (1). p 165175.

Medeiros, R. K. S et al. (2015) Modelo de validação de conteúdo de Pasquali nas pesquisas em Enfermagem. Rev Enferm Ref; (4) p 127-35.

Minayo, M. C. S. (2010). O desafio do conhecimento: pesquisa qualitativa em saúde. (12. ed.), Hucitec.

Ministério da Saúde (2020). Secretaria de Atenção Especializada à Saúde/SAES Departamento de Atenção Hospitalar, Domiciliar e de Urgência/DAHU Coordenação Geral de Atenção Hospitalar e Domiciliar/CGAHD. Manual de Cuidados Paliativos. São Paulo: Hospital Sírio Libanês.

Pacheco, L. S. P et al (2020). O processo de comunicação eficaz do enfermeiro com o paciente em cuidados paliativos. Rev Research, Society and Development, 9 (8). p 1-15.

Passarelles, D. M. A.; Rios, A. A.; Santana, R. F. (2019). Diagnósticos de enfermagem em cuidados paliativos oncológicos: revisão integrativa. Rev Enfermería Global. (55). p 590-600.

Perondi, B. L. B et al. (2019). A enfermagem nos cuidados paliativos de criança com leucemia linfoide aguda terminal. Revista Ciências da Saúde e Educação IESGO; (1) 1. p 29-36.

Queiroz, T. A et al (2018). Cuidados paliativos ao idoso na terapia intensiva: olhar da equipe de enfermagem. Rev Texto Contexto Enfermagem; 27 (1). p 1-10. Rapanos, B. M.; Oliveira, E. C.; Ferreira, W. F. S. (2020). Percepções e conflitos dos acadêmicos de enfermagem sobre cuidados paliativos. Revista de Atenção à Saúde; 18 (65). p 59-75.

Santos, D. J. L. D. L. et al. (2019). O enfermeiro e os cuidados paliativos proporcionados ao idoso terminal internado em UTI. Brazilian Journal of health Review. p 1095-1104.

Santos, J. B. S. et al. (2017). Assistência Integral De Enfermagem Aos Pacientes Em Cuidados Paliativos. Revista Saúde; (11) 1. p 1-16.

Silva, A.F. et al. (2015). Cuidados paliativos em oncologia pediátrica: percepções, saberes e práticas na perspectiva da equipe multiprofissional. Rev Gaúcha Enfermagem; 36 (2): 56-62.

Silva, R.S. et al. (2017). Construção e validação de diagnósticos de enfermagem para pessoas em cuidados paliativos. Rev. Latino-Am. Enfermagem. 25:e2914. p. 1-9.

Silveira, N. R. et al. (2016). Cuidado paliativo e enfermeiros de terapia intensiva: sentimentos que ficam. Revista Brasileira de Enfermagem; (69) 6. p 1-8. 